\title{
Noise sources in silver halide volume diffuse-object holograms
}

\author{
Antonio Fimia \\ Luis Carretero \\ Rosa Fuentes \\ Universidad de Alicante \\ Laboratorio de Optica \\ Departamento Interuniversitario de Optica \\ Apartado 99 \\ Alicante E-03080, Spain \\ E-mail: fimia@rm.cpd.ua.es
}

Augusto Beléndez, MEMBER SPIE
Universidad de Alicante
Departamento de Ingeniería de Sistemas y
Comunicaciones
Apartado 99
Alicante E-03080, Spain

\begin{abstract}
Dichromated gelatin has been established as the most frequently used recording material for the production of holographic optical elements. New applications are being found for photopolymers in previously unexplored areas such as holographic interconnect systems. However, photographic emulsion from the beginning has been and continues to be the most used holographic recording material. This is due to its relatively high sensitivity and ease of processing, the availability of improved processing chemistries and commercial films, and the repeatibility of the results. We analyze different sources of noise in photographic emulsions (such as intermodulation noise, noise gratings, and nonlinear noise) and the influence of the photochemical process on those noise sources. Bleached emulsions using rehalogenating and solvent processes are considered, and silver-halide-sensitized gelatin is discussed as a medium for transmission holograms. New developers and new noise models are presented on the supposition that the nonlinear response of the recording material is due to the photochemical process.
\end{abstract}

Subject terms: holography; holographic recording materials; diffuse-object holograms; diffraction efficiency; signal-to-noise ratio; bleached emulsion; silverhalide-sensitized gelatin.

Optical Engineèring 34(4), 1108-1115 (April 1995).

\section{Introduction}

Silver halide emulsions have long been used as a medium for recording volume phase holograms. This is due to their high sensitivity and ease of processing, the availability of improved processing chemistries and commercial films, and the repeatibility of the results. Unfortunately, while the resulting phase holograms have high diffraction efficiency, this is usually accompanied by an increase in the noise and a consequent reduction in image quality. Over the last 30 years many papers have been published in which different types of photochemical processing for silver halide emulsions and the experimental results obtained when using them have been presented and analyzed. Thus, holography has served as a technique with which to optimize different types of photochemical processing, and if high diffraction efficiencies are obtained, processing is optimized. Nevertheless, one char-

Paper 23054 received May 16, 1994; revised manuscript received Oct. 11, 1994; accepted for publication Oct. 12, 1994. This paper is a revision of a paper presented at the SPIE conference on Practical Holography VIII, Feb. 1994, San Jose, Calif The paper presented there appears (unrefereed) in SPIE Proceedings Vol. 2176. (c) 1995 Society of Photo-Optical Instrumentation Engineers. 0091-3286/95/\$6.00 acteristic of all silver halide holograms, no matter what the processing used, is the level of noise that degrades the quality of the holographic image.

The first papers in the field of holography dedicated to the analysis of noise sources focused on scattering in holographic gratings and on intermodulation noise in diffuse objects. These studies were based on the belief that there exists a linear relation between exposure and the index modulation obtained during the bleaching process. At the same time, several different chemical compositions were developed to be used as bleaches in an attempt to suppress or minimize the sources of noise. Among these we find reversal processes using solvent bleaches ${ }^{1}$ and rehalogenating processes ${ }^{2}$ using tanning agents in the developer and bleaching baths. ${ }^{3}$ In both cases, the goal was to obtain high diffraction efficiency and high signal-to-noise ratio.

From a theoretical point of view, the study done by Upatnieks and Leonard ${ }^{4}$ offered the first theoretical model, and this model, together with the one presented by Kogelnik ${ }^{5}$ for holographic gratings, made it possible to analyze and study intermodulation noise in diffuse-object holograms. It was assumed that the behavior of intermodulation noise was sta- 
tistical in nature and that the variations in the average refraction index were proportional to exposure. This model proved the importance of the beam ratio for the signal-tonoise ratio of a diffused object. More recent studies have shown that there exist noise sources due to the granular nature of emulsions, which are called noise gratings. ${ }^{6}$ These have been carefully studied both theoretically and experimentally over the last few years. ${ }^{7}$ These noise gratings are one of the many sources of noise in the image of diffuse-object holograms, and they are influenced by the state of polarization of the reconstruction wave, the processing used, and exposure. $^{8}$

In this paper we attempt to analyze the different sources of noise in diffuse objects, using as a starting point the current knowledge that we have of photochemical processing with photographic emulsions. We have considered rehalogenating bleaches with and without a fixation step, solvent bleaches without a fixation step, and silver-halide-sensitized gelatins.

\section{Bleached Silver Halide Diffuse-Object Holograms}

\subsection{Rehalogenating Bleaches with a Fixation Step}

The first holograms of dielectric diffuse objects were obtained using processes in which the emulsion was developed, fixed, and then bleached. These are known as rehalogenation processes. As part of the process, the exposed areas in which there are traces of metallic silver are subsequently processed, and as a result they once again have silver halide in them. This generates a clear difference in the refractive index of the silver halide-gelatin area and the area where there is only gelatin.

While it is true that diffraction efficiency is fundamental from the perspective of holographic gratings and lenses, it is also true that the signal-to-noise ratio (SNR) is basic when dealing with diffuse-object holograms. In phase diffuseobject holograms recorded in silver halide emulsions processed with a rehalogenating bleach with a fixation step, noise gratings do not exist, because this processing technique introduces considerable emulsion thickness change. Due to this, the principal sources of noise that we must consider are scattering and the intermodulation noise caused by the selfinterference of light from the extended object.

Diffuse-object holograms were recorded in Agfa-Gevaert $8 E 75 \mathrm{HD}$ emulsion. Several different beam ratios were used, and the exposures were made with 633-nm radiation from a $\mathrm{He}-\mathrm{Ne}$ laser. The object used was a $2-\mathrm{cm} \times 2-\mathrm{cm}$ square with a central opaque zone measuring $1 \mathrm{~cm} \times 1 \mathrm{~cm}$. Figure 1 shows a schematic representation of the geometry used in our experiments. The distance of the object from the recording medium was $30 \mathrm{~cm}$, and the angle between the object and the reference beams was $40 \mathrm{deg}$. The reference-to-object beam ratios used were $K=1,5,10$, and 25 .

Once the plate was processed, holograms were replayed by the conjugate of the collimated reference beam, and the diffracted output beam formed the real image of the square. The following equation was used to calculate the signal-tonoise ratio:

$\mathrm{SNR}=\frac{I_{1}+I_{2}}{2 I_{0}}$,

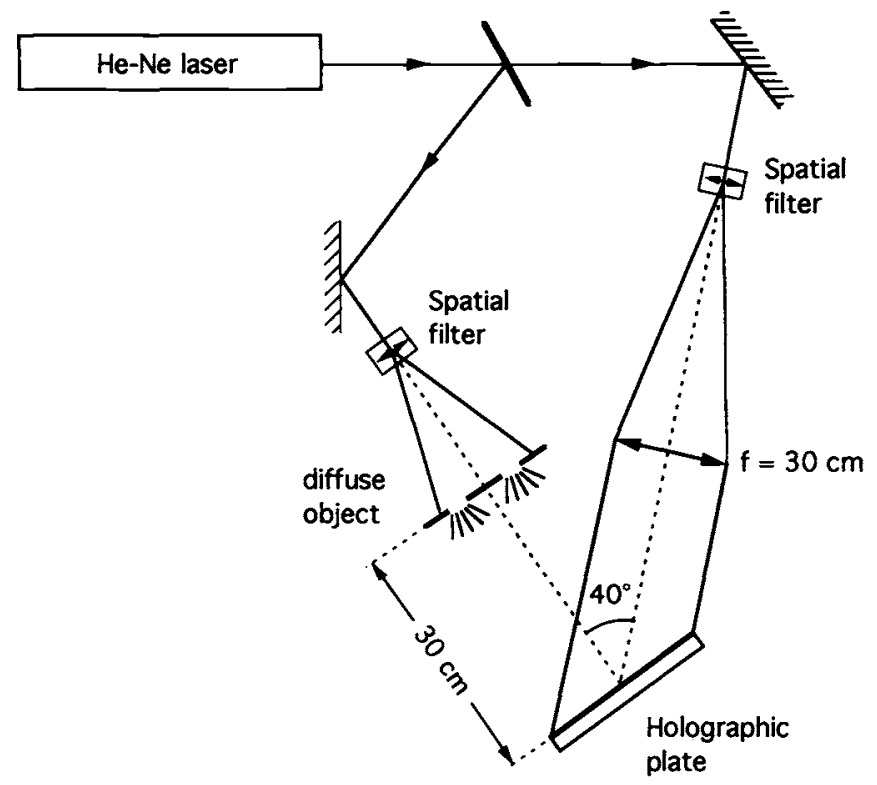

Fig. 1 Experimental setup used for recording of holograms.

where $I_{1}$ and $I_{2}$ are the intensity levels on the two sides of the square and $I_{0}$ is the intensity level in the central region.

In Fig. 2 we show the diffraction efficiency and the SNR for three different developers: D-8, D-19, and D-76, each of which is characterized by a different slope of the $D-\log E$ curve. In all three, the same potassium ferrocyanide and potassium bromide bleaching agent was used. Figure 3 shows the diffraction efficiency when the D-8 developer is used with different beam ratios. As is clearly seen, developers with only a slight slope (D-76) have a low diffraction efficiency and better SNR than those with a steep slope (D-8). Likewise, when the beam ratio $K$ increases, the SNR also increases, while at the same time the diffraction efficiency decreases.

Because these processes present a very high noise level, Lamberts and Kurtz ${ }^{1}$ proposed the use of solvent-type bleaches. These produce a clear improvement in the SNR without causing a decrease in the diffraction efficiency. It is a well-known fact that this bleaching has the advantage that the relief image resulting from local tanning by the developer depresses the modulation transfer function at low spatial frequencies and produces a reduction in intermodulation noise. It is worth noting that in all cases, the SNR increases significantly for high exposures.

\subsection{Solvent Bleaches}

These types of processing are characterized by the bleaching action taking place in the exposed area. They are not submitted to a fixing step, and therefore the metallic silver grains dissolve and compensate the optic path between the exposed and unexposed areas due to the thickness variation that takes place. The bleach converts the developed silver into $\mathrm{Ag}^{+}$, which passes into the solution, leaving a phase hologram made up of the unexposed silver halide grains. Solvent bleaches produce good diffraction efficiencies and low scattering values, because the size of the unexposed silver halide grains is not affected significantly by the processing solutions. Figure 4 gives an example of the diffraction efficiency and SNR for a typical solvent process (R-9); see Table 1. As can 


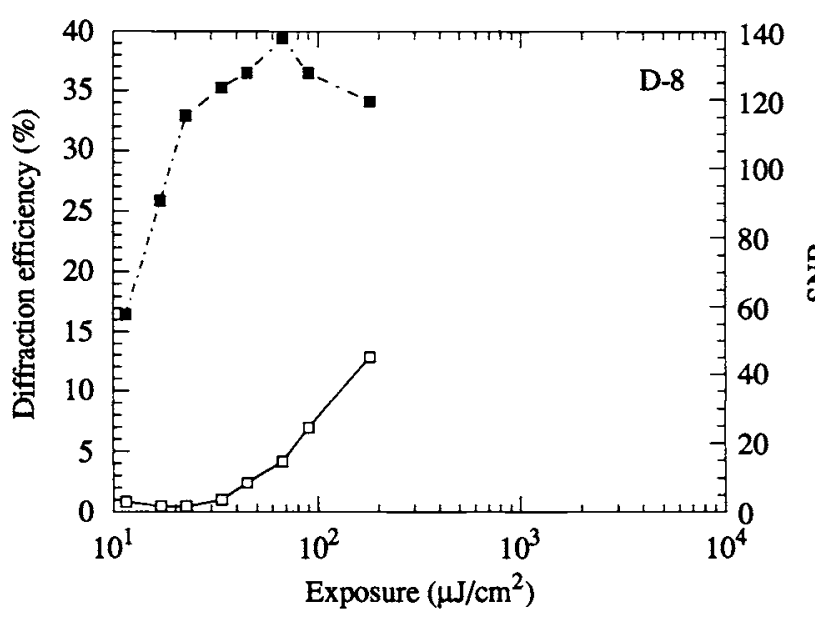

(a)

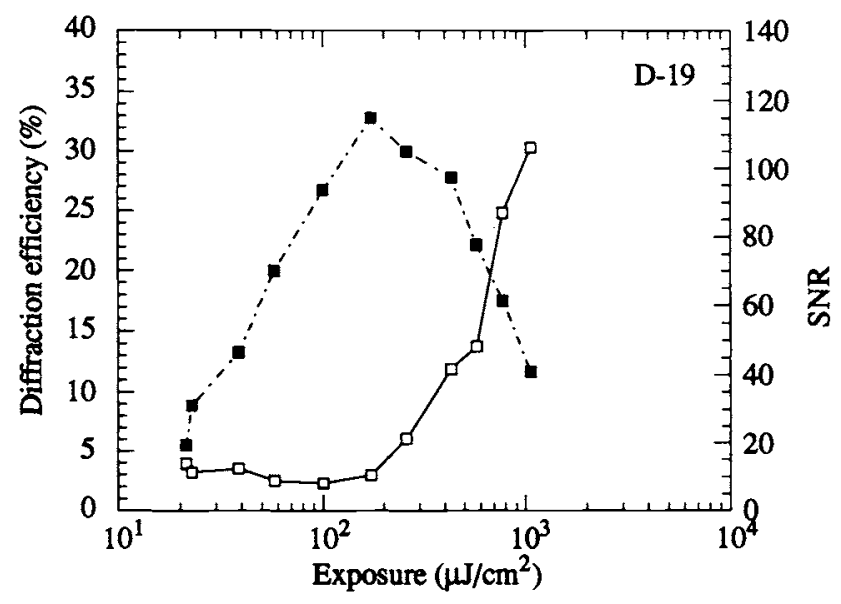

(b)

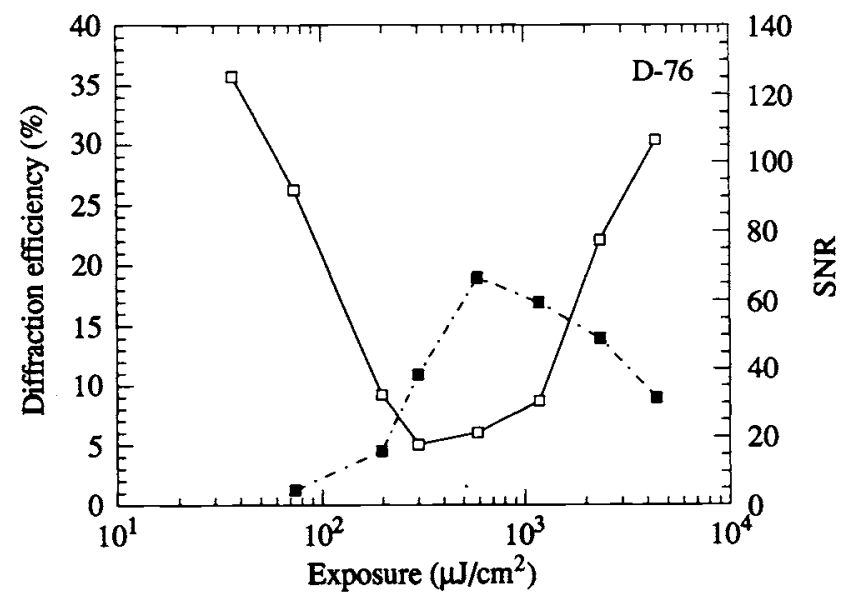

(c)

Fig. 2 Diffraction efficiency ( $\square$ ) and SNR ( $\square$ ) for three different developers and for a beam ratio $K=5$ : (a) $D-8$, (b) D-19, and (c) D-76.

be seen, the diffraction efficiency is lower than when rehalogenation with fixation is used, but the SNR is clearly higher.

\subsection{Rehalogenating Bleaches without a Fixation Step}

The chemical processes involved in this type of bleaching have been the cause of much controversy. Recently studies have been done that confirm the action of these bleaches. ${ }^{9}$ In these processes, after development, the emulsion is rehalogenated. First there is diffusion of the silver ions, and then the formation of silver ions and bromide ions takes place due to the solubility of the unexposed silver bromide grains in the bleached bath. This brings about a reordering of the material and consequently an index modulation between the exposed and unexposed areas. The finite, though small, solubility action in these processes produces small variations in thickness. Two types of bleach baths were used in these experiments. One was R-10, and the other was EDTA. Figure 5 shows the diffraction efficiency and the SNR for this type of processing. As can be seen, we obtain good diffraction efficiency and excellent SNR. This is an improvement over the results obtained with solvent processing. Thus it is this type of processing that is most frequently used today.

In a recent study we experimentally analyzed other sources of noise in diffuse-object holograms, such as noise gratings. We found that these noise gratings, recorded during formation of the hologram, produce a drop not only in the diffraction efficiency but also in the noise, and that they have a significant influence on the final characteristics of diffuseobject holograms.

\subsection{Theoretical Models}

All of the previous experimental results are difficult to derive from a theoretical model, given the great number of parameters that come into play: exposure, developing and bleaching solutions, type of processing, linearity of the developer, slope, beam ratio, etc. Upatnieks and Leonard were able to use their model to show that diffraction efficiency and SNR are a function of the beam ratio and at the same time point out the importance of the effects of volume when attempting to improve the SNR.

From the perspective of the coupled-wave theory, and assuming that an object is a set of $N$ waves, Kostuk $^{10}$ attempts to create a model to show the relation between the SNR and the influence of the emulsion thickness. However, his results are also based on a linear relation between index modulation and exposure, and they are not compatible with the results obtained by Upatnieks and Leonard. There was also the problem of the limitations of the numerical calculation in the resolution of $N$ coupled waves. We have recently suggested the possibility of a nonlinear relationship between exposure and index modulation and have been able to show high SNRs with very high exposures. ${ }^{11}$ Nevertheless, none of these models takes into account all of the noise sources that are found when a diffuse object is stored. By considering the nonlinear behavior of the recording material in the case of diffuse-object holograms, better agreement between theoretical predictions and experimental results has been achieved. When the beam ratio is low, there is agreement between the nonlinear model and the experimental results, but when the beam ratio is large, we observed that the nonlinear model and the experimental results do not agree, because in this case the process is linear. Figure 6 shows the results of the measurements of SNR when the plates are immersed in oil as compared to when they are not immersed, using the nonlinear model. As can be seen, in this case the model more accurately predicts the experimental results obtained, because in this case we only analyzed noise caused 


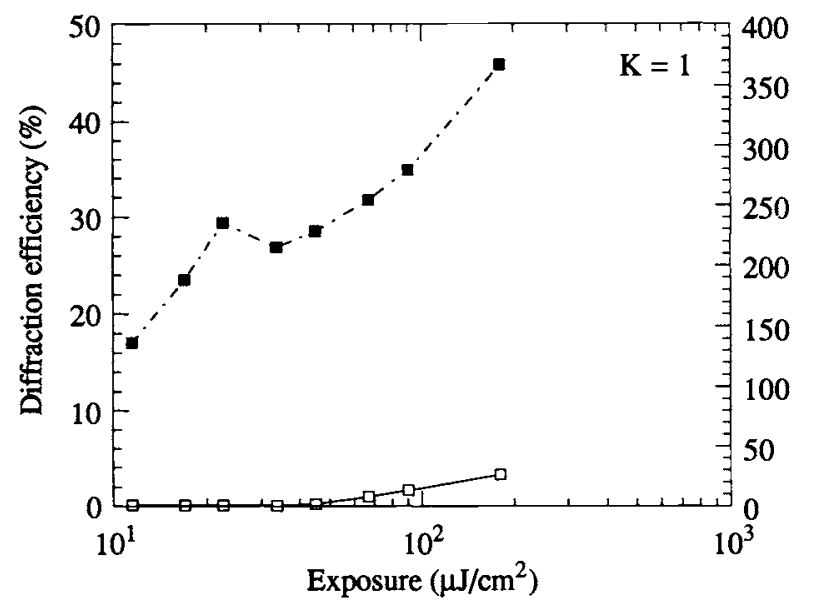

(a)

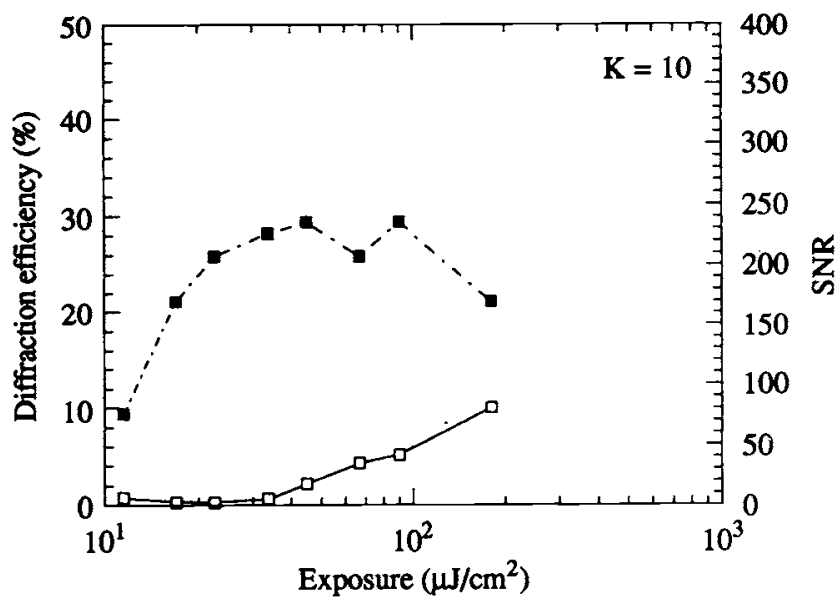

(c)

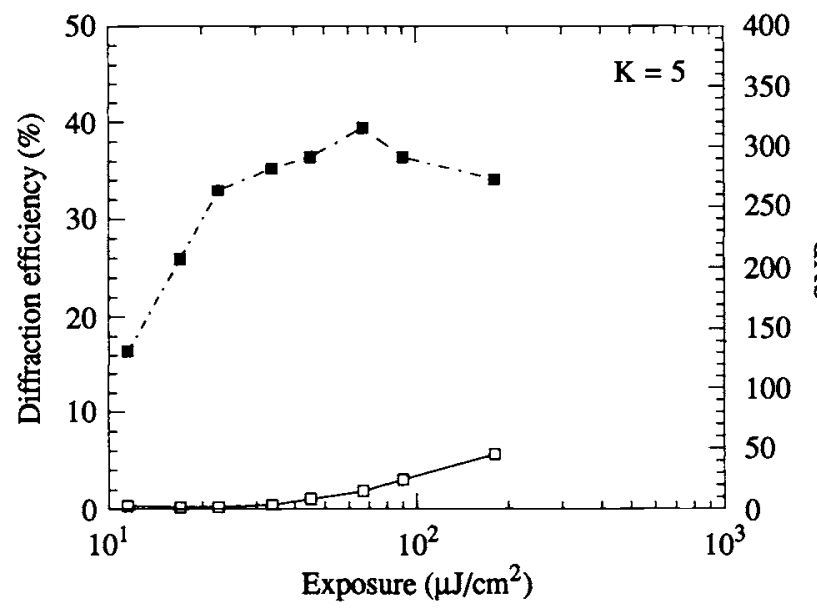

(b)

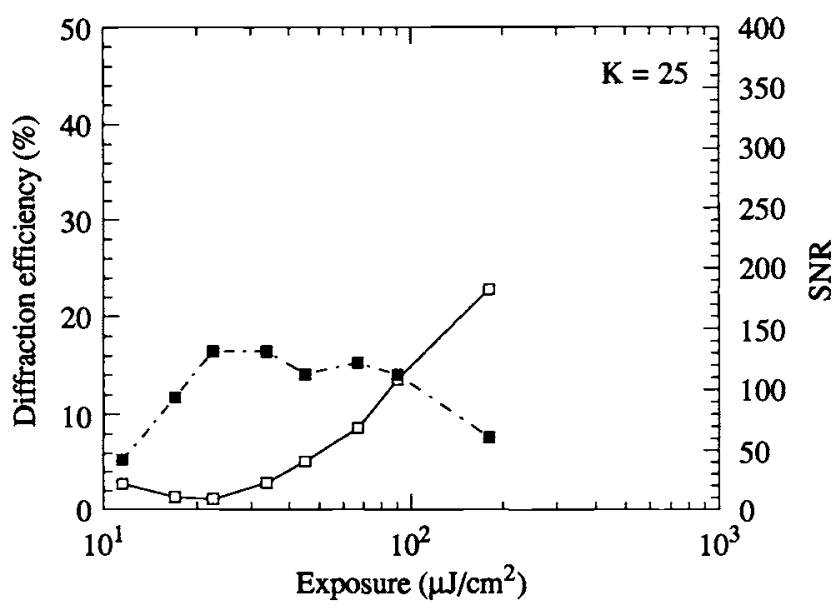

(d)

Fig. 3 Diffraction efficiency ( $(\square)$ and SNR ( $\square$ ) for different beam ratios $K$ when the D-8 developer is used: (a) $K=1$, (b) $K=5$, (c) $K=10$, and (d) $K=25$.

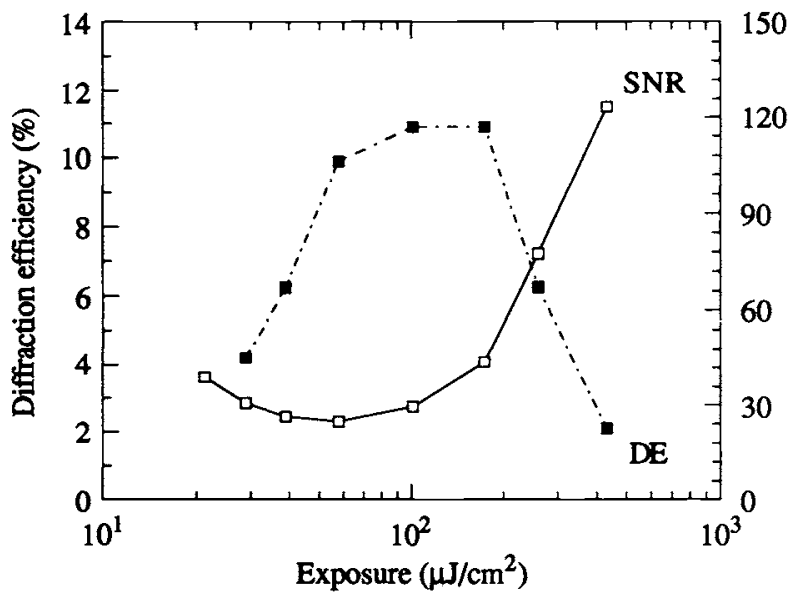

Fig. 4 Diffraction efficiency (DE) and SNR for a typical solvent process $(R-9)$. by index variation, inasmuch as when the plates are in oil the principal source of noise is intermodulation noise. These studies are important, given that they are related to cross talk in holographic interconnection systems used in optical computing, in which the models proposed up to now are linear. ${ }^{12}$

\section{Optimization Processes in Bleaches}

爱 The first conclusion which we can draw from an analysis of the historic antecedents that we have is that all optimization processes should combine the correct selection of the developer with the correct selection of the bleaching solution. In Tables 1 and 2 we show possible developing and bleaching solutions that provide high energy sensitivity while at the same time achieving excellent SNRs and high diffraction efficiency. As can be seen, an ascorbic acid and phenidone developer was used along with a rehalogenating bleach without a fixation step. The concentration of bromide in this solution was adjusted in order to obtain a significant decrease in scattering. Figure 4 shows our results for diffraction ef- 
Table 1 Bleach-bath formulas.

\begin{tabular}{lrrrr}
\hline & $\mathrm{R}-9$ & $\mathrm{R}-10$ & $\mathrm{Fe}$ & EDTA \\
& & & & \\
\hline Potassium bromide $(\mathrm{K} \mathrm{Br})$ & & $35 \mathrm{~g}$ & $7 \mathrm{~g}$ & $30 \mathrm{~g}$ \\
$\begin{array}{l}\text { Potassium ferrocyanide }\left(\mathrm{K}_{3} \mathrm{Fe}(\mathrm{CN})_{6}\right) \\
\text { Potassium dichromate }\left(\mathrm{K}_{2} \mathrm{Cr}_{2} \mathrm{O}\right)\end{array}$ & $2 \mathrm{~g}$ & $2 \mathrm{~g}$ & & \\
Sulfuric acid $\left(\mathrm{H}_{2} \mathrm{SO}_{4}\right)$ & $10 \mathrm{cc}$ & $10 \mathrm{cc}$ & & $10 \mathrm{cc}$ \\
Ferric sulfate $\left(\mathrm{Fe}_{2}\left(\mathrm{SO}_{4}\right)_{3}\right)$ & & & & $30 \mathrm{~g}$ \\
EDTA disodium salt & & & & $30 \mathrm{~g}$ \\
Water to make & 1 liter & 1 liter & 1 liter & 1 liter \\
\hline
\end{tabular}

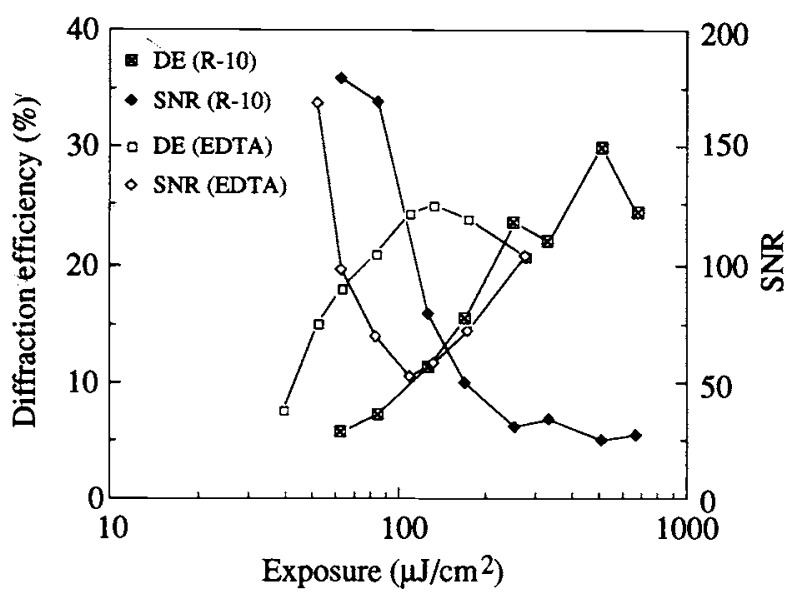

Fig. 5 Diffraction efficiency (DE) and SNR for R-10 and EDTA rehalogenating bleach baths.

ficiency and SNR when using R-10 and EDTA. The SNR is clearly improved when using this last bleach, and no loss of diffraction efficiency takes place.

Therefore, the first conclusion that can be drawn is that it is possible to increase the SNR without decreasing diffraction efficiency. However, we must first have a better understanding of the SNRs separately so that we can analyze the improvement obtained in the SNR.

In Figure 7 we show the angular response of (a) the noise and (b) the diffraction efficiency when the reconstruction angle is changed. The noise was measured as the ratio between the minimum light intensity in the central square on the reconstructed object and the incident light intensity. As can be seen in Fig. 7, there is a low point exactly at the value corresponding to the Bragg angle, which is typical when noise gratings are present. An analysis of the transmitted zero order leads us to the same conclusion [Fig. 8(a)]. We find that if we compare the diffraction efficiency of the noise found in rehalogenating processes with and without a fixation step, the noise level is lower in the latter case when the beam ratio is the same than it is when a fixation step is done, in spite of the fact that in the rehalogenating process with fixation there are no noise gratings in the reconstruction direction due to thickness variations.

By analyzing the reflection ratio, we see that there are also reflection noise gratings and they surely play a role in the

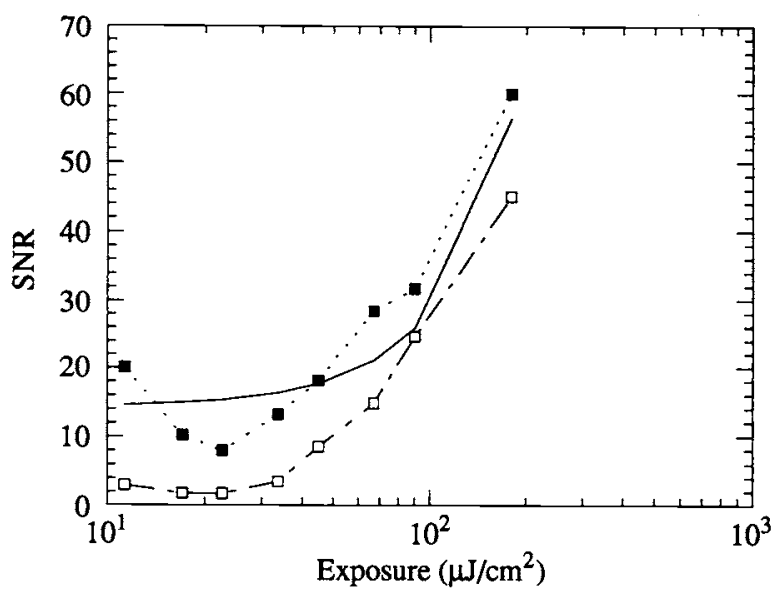

Fig. 6 SNR as a function of exposure for $K=5$ : index-matching plates ( $\square$ ), non-index-matching plates (ㄷ), and nonlinear model ( - ). The plates were developed with $\mathrm{D}-8$, and were fixed and bleached in a bath that contained potassium bromide $(7 \mathrm{~g})$, potassium ferrocyanide $(8 \mathrm{~g})$, and distilled water to make 1 liter

Table 2 Developer formula.

\begin{tabular}{ll} 
PAAAC Developer & \\
Sodium Carbonate & $120 \mathrm{~g}$ \\
Ascorbic Acid & $18 \mathrm{~g}$ \\
Phenidon & $0.5 \mathrm{~g}$ \\
Distilled water to make & 1 liter \\
& \\
\hline
\end{tabular}

decrease of both diffraction efficiency and noise [Fig. 8(b)]. Measurements taken after changing the state of polarization of the measurement beam show the disappearance of the low points mentioned previously, which are typical of the noise gratings. As far as we know, these effects have not been reported in the literature prior to this for cases in which noise gratings appear in diffuse-object holograms. ${ }^{13}$ Additionally, as can be seen in Fig. 8(b), the peaks corresponding to the decrease in diffraction efficiency also appear in the measurements of the noise ratio.

This second conclusion can be summed up by saying that in spite of the appearance of more noise gratings when rehalogenation processes with a fixation step are used than when these same processes without a fixation step are used, the levels of noise are lower for the processes without fixation.

The possible solvent action of the EDTA-type rehalogenation bleaches and the use of developers without agents that give rise to oxidation products bring about the optimization and improvement of SNR and diffraction efficiency.

\section{Diffuse-Object Holograms in Silver-Halide-Sensitized Gelatin}

In all of the processes analyzed above, the recording material has always been granular in nature. Silver-halide-sensitized gelatins (SHSG) have proven to be an alternative to bleaching processes and dichromated gelatins for transmission holographic gratings. 


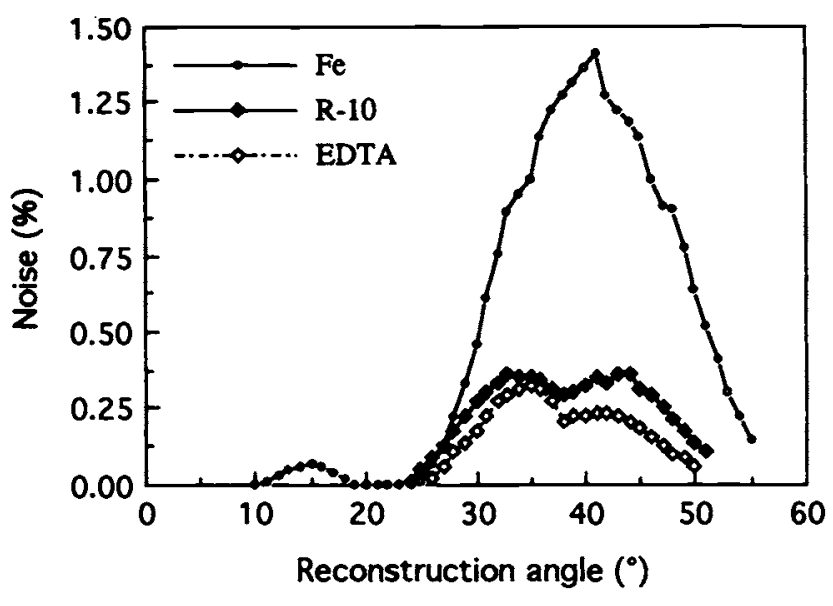

(a)

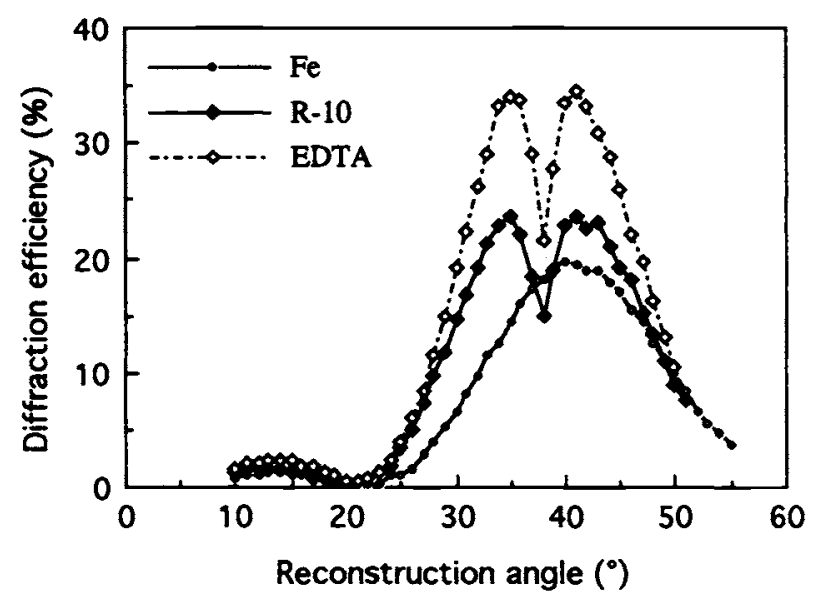

(b)

Fig. 7 Angular response when $\mathrm{R}-10$ and EDTA rehalogenating bleach baths without fixing and Fe bleach baths were used: (a) noise and (b) diffraction efficiency.

The use of SHSG is a hybrid processing method that combines the energetic and spectral sensitivity of photographic emulsion with the high diffraction efficiency and low noise of dichromated gelatin. ${ }^{14}$ In SHSG processing, the developed silver of the photographic emulsion is converted through bleaching, fixing, washing, and dehydrating in isopropanol baths into a refractive-index modulation of the gelatin, forming a phase hologram. Basically, once the photographic emulsion has been exposed, it is developed and bleached. Due to this bleaching action, the developed silver is oxidized to $\mathrm{Ag}^{+}$, whereas the $\mathrm{Cr}^{+6}$ ion is reduced to $\mathrm{Cr}^{+3}$ during the same bleaching wash. In this way the $\mathrm{Cr}^{+3}$ ion is linked to the gelatin chains in the vicinity of the oxidized silver grains, achieving variation in hardening between the exposed and unexposed zones of the emulsion. Water developing creates an initial refractive-index modulation due to swelling, forming a strong volume hologram. This modulation is further amplified by the final alcohol developing. In a recent series of articles, ${ }^{15,16}$ some little-known models for the formation of latent images using this type of processing have been discussed. In Table 3, an example of the processing used in our laboratory is presented. ${ }^{14}$

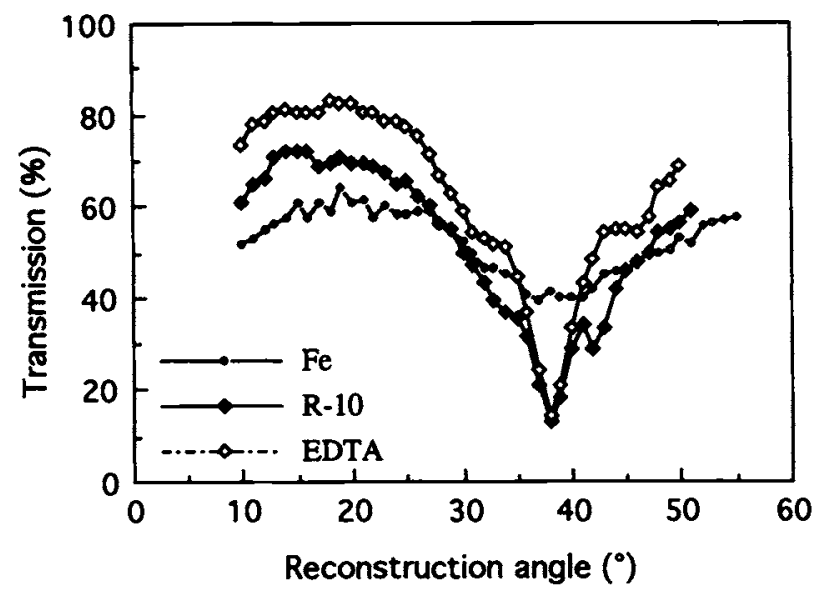

(a)

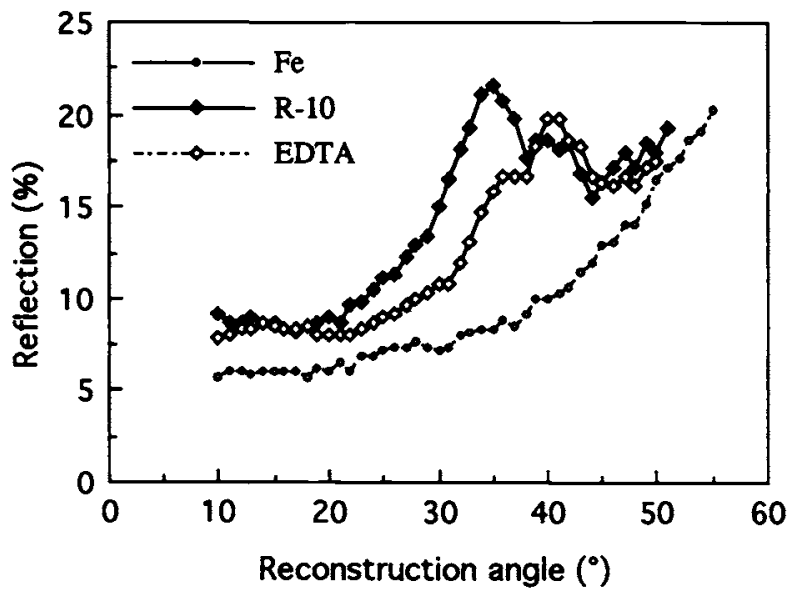

(b)

Fig. 8 (a) Transmission and (b) reflection when the reconstruction angle is changed. R-10 and EDTA rehalogenating bleach baths without fixing and $\mathrm{Fe}$ bleach baths were used.

Experiments were carried out with Agfa 8E75 HD plates using a He-Ne laser. A series of holograms was made. Exposures ranged from 4 to $50 \mu \mathrm{J} / \mathrm{cm}^{2}$, and the reference-toobject beam ratio was 5 . The use of this hybrid processing for the storage of diffuse-object holograms produced the results presented in Fig. 9. Although good SNRs are not achieved in the linear area of the developer, we see that it is possible to obtain SNRs near 70 with diffraction efficiencies of $28 \%$ for diffuse objects when the beam ratio is $K=5$. These results have not been achieved with any other material.

Finally, in a recent research study done with diffuse-object holograms ${ }^{15}$ we found that current models for the formation of latent images cannot completely account for the storage of information in SHSG holograms. These results show the need to modify existing models in order to be able to explain all the experimental results obtained with this type of processing.

\section{Conclusions}

The progress that has been made in the analysis and optimization of bleaches has led to an improved in image quality 
Table 3 Processing schedule for SHSG.

\section{STEP}

1.- Develop with PAAAC for 4 minutes.

2.- Rinse in running water for 1 minute

3.- Bleach in a modified R-10 solution for 30 seconds after the plate has cleared at $50^{\circ} \mathrm{C}$

4.- Rinse in running water for 30 seconds.

5.- Soak in fixer F-24 for 2 minutes.

6.- Wash in running water for 10 minutes.

7.- Dehydrate in $50 \%$ isopropanol for 3 minutes.

8.- Dehydrate in $90 \%$ isopropanol for 3 minutes.

9.- Dehydrate in $100 \%$ isopropanol for 3 minutes at $50^{\circ} \mathrm{C}$.

10.- Dry in vacuum chamber.

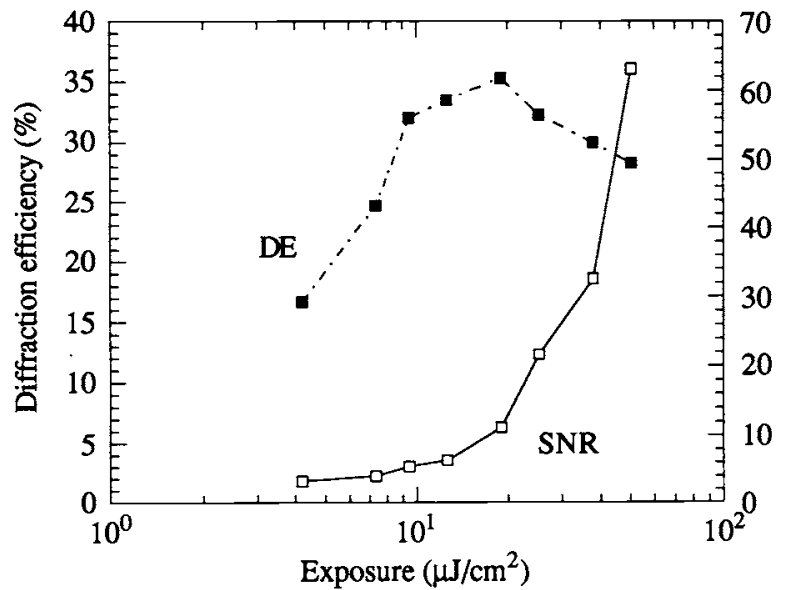

Fig. 9 Diffraction efficiency and SNR for silver-halide-sensitized gelatin diffuse-object holograms.

in diffuse-object holograms. The relation between the SNR and the diffraction efficiency cannot be explained with the models we have. This optimization is fundamentally based on the correct selection of the concentrations and composition of the developing and bleaching agents in each step of the process.

The inclusion of noise gratings as a new source of noise in diffuse-object holograms and the influence of these gratings on the final SNR present new perspectives for the analysis of noise in holography and at the same time allow us to obtain more information about the behavior of photochemical processes involved in bleaching.

Rehalogenating processes without a fixation step produce the best SNR for a specific diffraction efficiency and a constant beam ratio. However, hybrid processes, such as the ones used with silver-halide-sensitized gelatine and the "eastwest" process proposed by Phillips, ${ }^{16}$ are undoubtedly the ones that in the near future will produce excellent levels of SNR and diffraction efficiency.

Finally, we wish to mention that even though we have concentrated on the analysis of diffuse objects in this study, a large portion of the experimental results we have obtained are of great importance in the generation of interconnection holographic systems in which many object beams are also stored.

\section{Acknowledgments}

Part of this work was supported by the Direcció General d'Ensenyaments Universitaris i Investigació de la Generalitat Valenciana, Spain (project GV-1165/93).

\section{References}

1. R. L. Lamberts and C. N. Kurtz, "Reversal bleaching for low flare light in holograms," Appl. Opt. 10, 1342-1347 (1971).

2. R. L. van Renesse and F. A. J. Bouts, "Efficiency of bleaching agents for holography,"' Optik 38, 156-168 (1973).

3. N. J. Phillips, A. A. Ward, R. Cullen, and D. Porter, "Advances in holographic bleaches," Photogr. Sci. Eng. 24, 120-124 (1980).

4. J. Upatnieks and C. Leonard, "Efficiency and image contrast of dielectric holograms," J. Opt. Soc. Am. 60, 297-305 (1970).

5. H. Kogelnik, "Coupled wave theory for thick hologram gratings," Bell Syst. Tech. J. 48, 2909-2947 (1969).

6. R. R. A. Syms and L. Solymar, "Noise gratings in photographic emulsions," Opt. Commun. 43, 107-110 (1982).

7. L. Carretero, A. Beléndez, and A. Fimia, "Holographic noise gratings for analysing and optimizing photochemical processings in bleached silver halide emulsions," J. Mod. Opt. 40, 687-697 (1993).

8. A. Beléndez, L. Carretero, and I. Pascual, "Polarization influences on the efficiency of noise gratings recorded in silver halide emulsions,' Appl. Opt. 32, 7155-7163 (1993).

9. P. Hariharan, "Basic processes involved in the production of bleached holograms," J. Photogr. Sci. 38, 76-81 (1990).

10. R. K. Kostuk, "Comparison of models for multiplexed holograms," Appl. Opt. 28, 771-777 (1989)

11. A. Fimia, A. Beléndez, and L. Carretero, "Signal to noise ratio of nonlinearly recorded holograms of diffuse objects," Appl. Opt. 33, 76067610 (1994).

12. P. Asthana, G. P. Nordin, A. R. Tanguay, Jr., and B. R. Jenkins, "Analysis of weighted fan-out/fan-in volume holographic optical interconnections," Appl. Opt. 32, 1441-1469 (1993)

13. A. Fimia, R. Fuentes, and A. Beléndez, "Noise gratings in bleached silver halide diffuse-object holograms,', Opt. Lett. 19, 1243-1245 (1994).

14. A. Fimia, A. Beléndez, and I. Pascual, "Silver halide (sensitized) gelatin in Agfa-Gevaert plates: the optimized procedure," J. Mod. Opt. 38, 2043-2051 (1991).

15. A. Fimia, L. Carretero, I. Pascual, and A. Beléndez, "Diffuse-object holograms in silver halide sensitized gelatin," J. Mod. Opt. 41, 649653 (1994)

16. N. J. Phillips, "Bridging the gap between Soviet and western holography," SPIE Inst. Ser. IS8, 206-214 (1990).

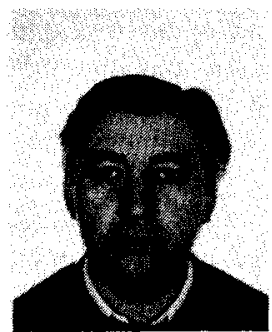

Antonio Fimia received his $\mathrm{PhD}$ degree in physics from the University of Alicante, Spain, in 1982. Since 1987 he has been a member of the Departamento Interuniversitario de Optica at the University of Alicante, where he is now a full professor. $\mathrm{He}$ has published over 60 papers on holographic recording materials, holographic optical elements, and computer-generated holograms. He has presented over 70 communications and participated in 14 research projects. His current research interests also include holography and intraocular lens design. He is a member of the OSA, EOS, ARVO, Holographic Group of SPIE, and SEDO. Since 1994 he has been the director of the Centro de Holografía, Fundación CIEA-CAM.

Luis Carretero received the $\mathrm{PhD}$ degree in physics from the University of Valencia (Spain) in 1994. He is presently a professor in the Optics Department at Alicante University. He has published over 15 papers on holographic recording materials and diffraction theory. $\mathrm{He}$ has presented over 20 communications to congresses. He has participated in three research projects. His research interests include holographic optical elements, diffraction theory, and image quality in holographic systems. 
Rosa Fuentes received the $\mathrm{PhD}$ degree in physics from the University of Valencia (Spain) in 1994. She is presently a professor in the Optics Department at Alicante University. She has published 13 papers on holographic recording materials and noise in holography. She has presented over 20 communications to congresses. She has participated in four research projects. Her current research interests include holography and holographic optical interconnectors.

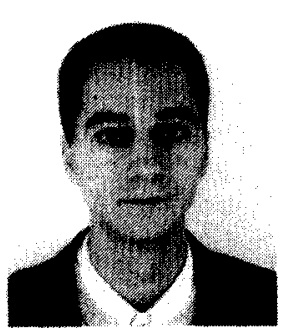

Augusto Beléndez received the $\mathrm{PhD}$ degree in physics from the University of $\mathrm{Val}$ encia (Spain) in 1990. From 1986 to 1991 he was a member of the Applied Physics Department at the Technical University of Valencia, and since 1991 he has been a professor of physics of the Systems Engineering and Communications Department of the University of Alicante, Spain. His research interests include holographic optical elements, holographic recording material, holographic optical interconnects, and diffraction theory. He has published more than 45 technical papers in various journals and has presented more than 50 papers at conferences and scientific meetings. He is a member of OSA, EOS, IEEE, SPIE's Technical Working Group on Holography, RSEF, and SEDO. 